\title{
Elevated fibrous sheath interacting protein 1 levels are associated with poor prognosis in non-small cell lung cancer patients
}

\author{
Yuqiang Mao ${ }^{1}$, Ran Xü ${ }^{1}$, Xiaoying Liu ${ }^{2}$, Wenjun Shi ${ }^{1}$, Yun Han ${ }^{1}$ \\ ${ }^{1}$ Department of Thoracic Surgery, Shengjing Hospital of China Medical University, Shenyang 110004, China \\ ${ }^{2}$ Department of Plastic Surgery, The First Hospital of China Medical University, Shenyang 110001, China \\ Correspondence to: Yun Han, email: hany@sj-hospital.org \\ Keywords: fibrous sheath interacting protein 1, non-small cell lung cancer, prognosis, TNM staging \\ Received: November 14, 2016 \\ Accepted: December 09, 2016 \\ Published: January 10, 2017
}

\section{ABSTRACT}

In this study, we examined the expression and prognostic value of fibrous sheath interacting protein 1 (FSIP1) in 202 non-small cell lung cancer (NSCLC) patients who underwent lung cancer resection at Shengjing Hospital of China Medical University. FSIP1 mRNA and protein expression were measured in NSCLC tissues and non-tumor adjacent tissues (NATs), and Harrell's concordance index (c-index) was used to evaluate the ability of FSIP1 to predict prognosis. FSIP1 mRNA and protein expression was higher in NSCLC tissues than in NATs. Survival analysis revealed the 5-year overall survival rate to be $35.4 \%$ in the FSIP1-positive group and $56.3 \%$ in the FSIP1negative group, and FSIP1-positive status was an independent prognostic factor for poor overall survival. The c-index value of FSIP1 for overall survival was greater than that of Ki67, and the addition of FSIP1 status increased the c-index value of the TNM staging system. These results suggest that evaluating FSIP1 status in addition to TNM stage during routine pathological examinations could improve prognostic predictions in NSCLC patients.

\section{INTRODUCTION}

Lung cancer is one of the most frequently diagnosed cancers and the leading cause of cancer-related death in China [1]. Although considerable advances have been made in surgery, adjuvant chemoradiotherapy, and targeted therapy, the prognosis for lung cancer remains poor [2]. This high mortality rate may be partly due to the lack of effective prognostic biomarkers. Currently, prognostic predictions are largely based on TNM staging. However, lung cancer patients at the same TNM stage may have different prognoses. New prognostic biomarkers are needed to more accurately identify high-risk lung cancer patients with poor prognoses.

Fibrous sheath interacting protein 1 (FSIP1) is a component of the microtubule- and dynein-rich fibrous sheath structure, which is necessary for flagellum function and sperm movement [3]. FSIP1 mRNA expression, which is low or undetectable in most normal tissues, is elevated in breast tumors [4]. Zhang et al. [5] reported that FSIP1 protein levels are also elevated in breast cancer tissues, and higher levels were associated with poorer prognosis in breast cancer patients. However, the role of FSIP1 in lung cancer remains unknown.

In this study, we measured FSIP1 expression in non-small cell lung cancer (NSCLC) and analyzed the association between FSIP1 and clinicopathological features. In addition, we evaluated the prognostic value of FSIP1 to determine whether it might be useful as a supplementary biomarker together with TNM stage in NSCLC patients.

\section{RESULTS}

FSIP1 expression is elevated in NSCLC

FSIP1 was measured in 20 NSCLC tissues and nontumor adjacent tissues (NATs) pairs using real-time PCR. FSIP1 expression was higher in NSCLC tissues compared to NATs in $90 \%(18 / 20)$ of these pairs (Figure 1A), and FSIP1 expression was significantly higher in NSCLC tissues than in NATs $(p<0.001$, Figure 1B). Similar results were obtained when protein levels were examined using western blots (Figure 1C). 
We then used immunohistochemistry to measure FSIP1 protein expression in 202 tissue pairs; 54\% (109/202) of these NSCLC tissues were FSIP1-positive and 46\% (93/202) were FSIP1-negative. In squamous carcinoma, FSIP1 expression was predominantly in tumor cell nucleus and also appearing in the cytoplasm. While in adenocarcinoma, FSIP1 expression was predominantly in the cytoplasm (Figure 2). Additionally, FSIP1 expression was higher in NSCLC tissues than in NATs (IS, 6.021 \pm 2.805 vs. $4.050 \pm 2.586$, respectively, $p<0.001$, Supplementary Table 1).

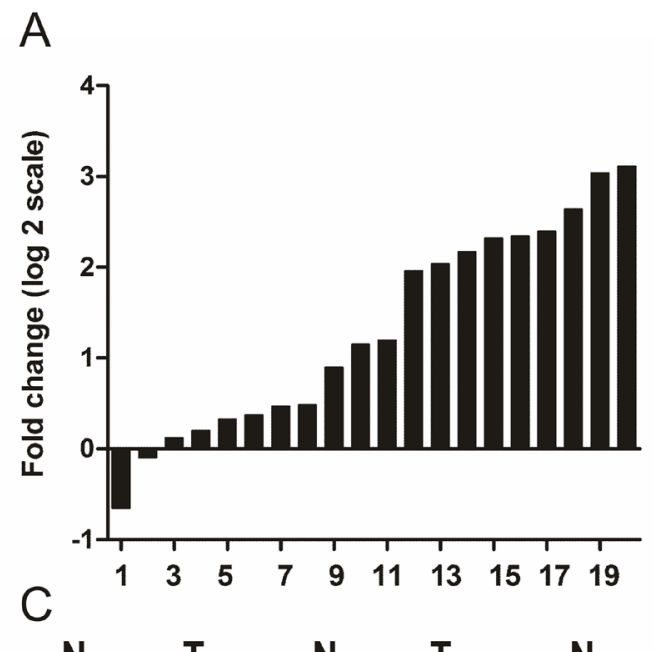

\section{Correlations between FSIP1 status and clinicopathological features}

We also examined associations between FSIP1 expression and clinicopathological features using the chi-square test. FSIP1-positive status was correlated with more advanced TNM stages $(p=0.042)$ and tended to be associated with more advanced $\mathrm{pN}$ categories ( $p=0.066$, Table 1$)$, although the latter association did not reach statistical significance. However, no significant relationships were found between FSIP1 expression and
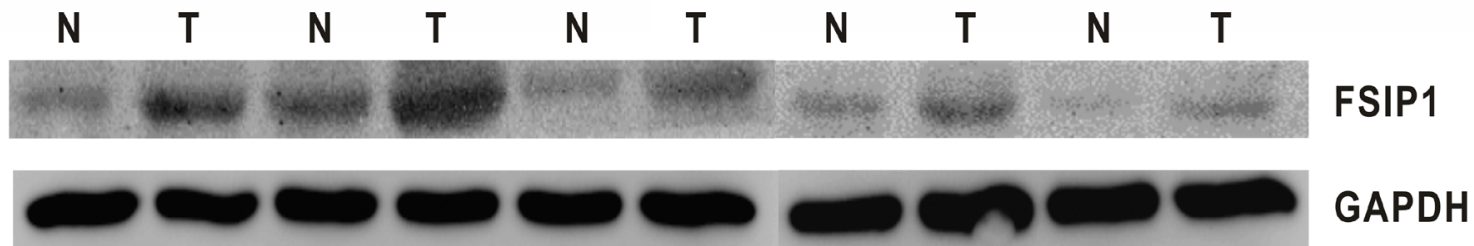

Figure 1: FSIP1 mRNA and protein expression are elevated in NSCLC. A. Data are presented as $\log _{2}$ of fold-change in FSIP1 in NSCLC tissues relative to non-tumor adjacent tissues. Each case was analyzed in triplicate and repeated three times. B. $\Delta$ CT values were used to compare the relative expression of FSIP1 in NSCLC tissues and non-tumor adjacent tissues. Data are shown as means \pm SD. Larger $\triangle \mathrm{CT}$ values indicate lower expression. C. Western blot indicated that FSIP1 protein was overexpressed in NSCLC tissues compared to non-tumor adjacent tissues.
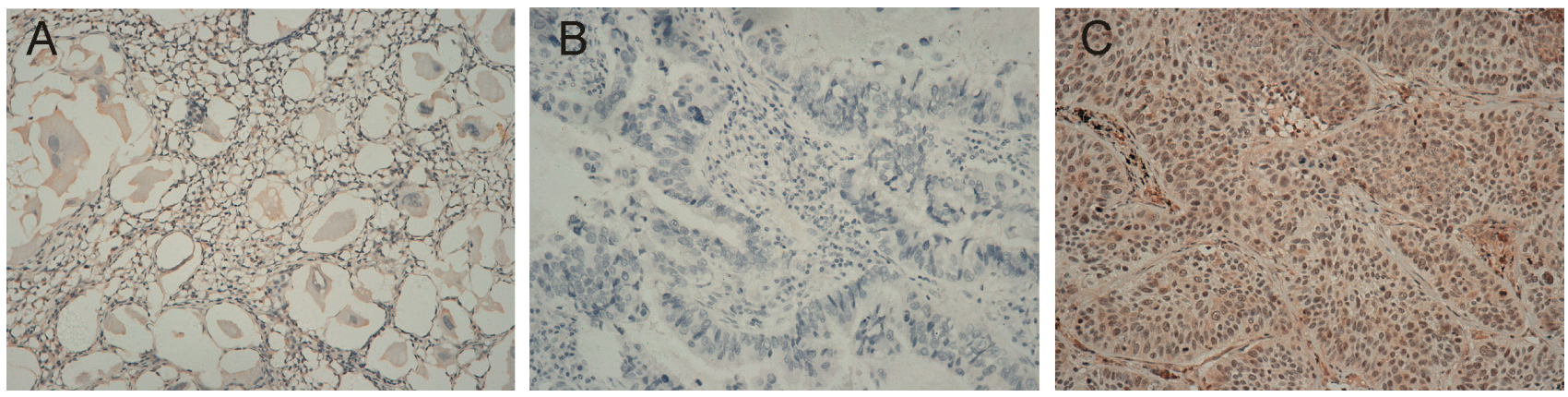

Figure 2: Immunohistochemical staining for FSIP1 in NSCLC tissues and non-tumor adjacent tissues. Magnification $\times$ 200. A. Non-tumor adjacent tissue (no stain). B. Negative FSIP1 staining in NSCLC tissues. C. Positive FSIP1 staining in NSCLC tissues. 
Table 1: Association between FSIP1 status and clinicopathological features

\begin{tabular}{|c|c|c|c|c|}
\hline \multirow{2}{*}{ Variables } & \multirow{2}{*}{ Number (\%) } & \multicolumn{2}{|c|}{ FSIP1 status } & \multirow{2}{*}{$P$ valu } \\
\hline & & FSIP1 (-) (\%) & FSIP1 (+) $(\%)$ & \\
\hline Sample size & $202(100)$ & $93(46.0)$ & $109(54.0)$ & \\
\hline Gender & & & & 0.175 \\
\hline Male & $96(47.5)$ & 49 (52.7) & $47(43.1)$ & \\
\hline Female & $106(52.5)$ & $44(47.3)$ & $62(56.9)$ & \\
\hline $\operatorname{Age}(y)$ & & & & 0.608 \\
\hline$>60$ & 119 (58.9) & $53(57.0)$ & $66(60.6)$ & \\
\hline$\leq 60$ & $83(41.1)$ & $40(43.0)$ & $43(39.4)$ & \\
\hline Histologic type & & & & 0.144 \\
\hline $\mathrm{AC}$ & $153(75.7)$ & $66(71.0)$ & $87(79.8)$ & \\
\hline $\mathrm{SC}$ & $49(24.3)$ & $27(29.0)$ & $22(20.2)$ & \\
\hline Differentiation & & & & 0.710 \\
\hline Well & $90(44.6)$ & 39 (41.9) & $51(46.8)$ & \\
\hline Moderate & $79(39.1)$ & 37 (39.8) & $42(38.5)$ & \\
\hline Poor & $33(16.3)$ & $17(18.3)$ & $16(14.7)$ & \\
\hline pT category & & & & 0.104 \\
\hline $\mathrm{T} 1$ & $82(40.6)$ & $40(43.0)$ & $42(38.5)$ & \\
\hline $\mathrm{T} 2$ & $89(44.1)$ & $45(48.4)$ & $44(40.4)$ & \\
\hline $\mathrm{T} 3$ & $18(8.9)$ & $5(5.40)$ & $13(11.9)$ & \\
\hline $\mathrm{T} 4$ & $13(6.4)$ & $3(3.20)$ & $10(9.2)$ & \\
\hline $\mathrm{pN}$ category & & & & 0.066 \\
\hline No & $129(63.9)$ & $67(72.0)$ & $62(56.9)$ & \\
\hline N1 & $22(10.9)$ & $9(9.7)$ & 13 (11.9) & \\
\hline N2 & $50(24.8)$ & $16(17.2)$ & $34(31.2)$ & \\
\hline $\mathrm{N} 3$ & $1(0.5)$ & $1(1.1)$ & $0(0)$ & \\
\hline Distant metastasis & & & & 0.223 \\
\hline Negative & $194(96.0)$ & $91(97.8)$ & $103(94.5)$ & \\
\hline Positive & $8(4.0)$ & $2(2.2)$ & $6(5.5)$ & \\
\hline TNM stage & & & & 0.042 \\
\hline I & $96(47.5)$ & $49(52.7)$ & $47(43.1)$ & \\
\hline II & $45(22.3)$ & $25(26.9)$ & $20(18.3)$ & \\
\hline III & $53(26.2)$ & $17(18.3)$ & $36(33.0)$ & \\
\hline IV & $8(4.0)$ & $2(2.2)$ & $6(5.5)$ & \\
\hline
\end{tabular}

Abbreviation, AC: adenocarcinoma; FSIP1: fibrous sheath interacting protein 1; SC: squamous carcinoma. 
other clinicopathological features such as gender, age, histologic type, differentiation, and pT category (all $p>0.05$, Table 1).

\section{FSIP1 expression is associated with poor prognosis}

The 5-year overall survival (OS) rate was 35.4\% in the FSIP1-positive patient group and $56.3 \%$ in the FSIP1-negative group ( $p<0.001$, Figure 3$)$. Furthermore, Cox multivariate analysis revealed that FSIP1-positive status was an independent prognostic factor for poor OS $(\mathrm{HR}=1.876,95 \% \mathrm{CI}=1.274-2.764, p=0.001$, Table 2).

We also examined the relationship between Ki67 expression status and prognosis. The 5-year OS rate was $39.2 \%$ in the Ki67-positive group and $59.2 \%$ in the Ki67-negative group ( $p=0.004$, Supplementary Figure 1). Cox multivariate analysis also indicated that Ki67- positive status was an independent prognostic factor for poor OS $(\mathrm{HR}=1.629,95 \% \mathrm{CI}=1.082-2.453, p=0.019$, Table 2).

\section{PFSIP1 has a higher prognostic ability}

We used the c-index method to evaluate the prognostic ability of different models. The c-index value of FSIP1 was greater than that of Ki67 (0.621 vs. 0.603), and the c-index value was greater for TNM and FSIP1 together than for TNM staging alone (0.735 vs. 0.716 , Figure 4).

\section{DISCUSSION}

Here, we measured FSIP1 expression in tissues from NSCLC patients. FSIP1 mRNA and protein expression were both higher in NSCLC tissues than NATs. We also found that FSIP1-positive status was correlated with more

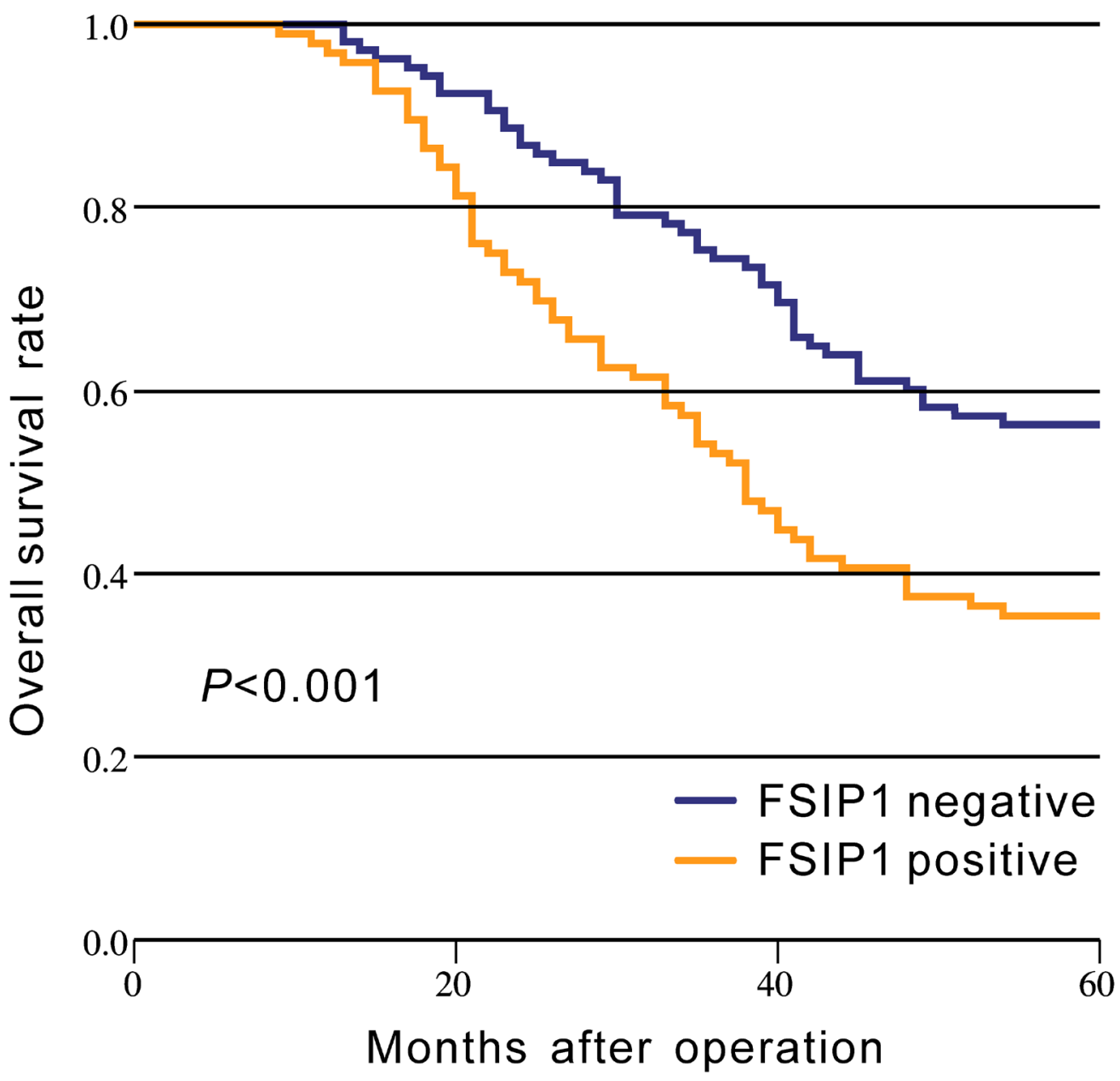

Figure 3: Kaplan-Meier analysis of overall survival based on FSIP1 status in 202 NSCLC patients. The 5-year overall survival rate in the FSIP1-positive group was lower than that in FSIP1-negative group $(p<0.001)$. 
Table 2: Univariate and multivariate analyses of overall survival in NSCLC patients

\begin{tabular}{|c|c|c|c|c|}
\hline \multirow{2}{*}{ Variable } & \multicolumn{2}{|c|}{ Univariate } & \multicolumn{2}{|c|}{ Multivariate } \\
\hline & HR (95\% CI) & $P$ & HR (95\% CI) & $\boldsymbol{P}$ \\
\hline Gender & & 0.537 & & \\
\hline Female & 1.000 & & & \\
\hline Male & $1.120(0.781-1.605)$ & & & \\
\hline Age (y) & & 0.174 & & \\
\hline$\leq 60$ & 1.000 & & & \\
\hline$>60$ & $1.297(0.892-1.885)$ & & & \\
\hline Histologic type & & 0.084 & & \\
\hline $\mathrm{AC}$ & 1.000 & & & \\
\hline $\mathrm{SC}$ & $0.669(0.424-1.055)$ & & & \\
\hline Differentiation & & 0.486 & & \\
\hline Well & 1.000 & & & \\
\hline Moderate & $1.256(0.846-1.863)$ & & & \\
\hline Poor & $1.237(0.736-2.078)$ & & & \\
\hline pT category & & $<0.001$ & & 0.038 \\
\hline $\mathrm{T} 1-\mathrm{T} 2$ & 1.000 & & 1.000 & \\
\hline $\mathrm{T} 3-\mathrm{T} 4$ & $2.198(1.411-3.424)$ & & $1.624(1.028-2.563)$ & \\
\hline $\mathrm{pN}$ category & & $<0.001$ & & 0.006 \\
\hline Negative & 1.000 & & 1.000 & \\
\hline Positive & $2.253(1.556-3.240)$ & & $1.726(1.171-2.543)$ & \\
\hline Distant metastasis & & $<0.001$ & & $<0.001$ \\
\hline Negative & 1.000 & & 1.000 & \\
\hline Positive & $6.583(3.158-13.725)$ & & $3.920(1.829-8.400)$ & \\
\hline TNM stage & & $<0.001$ & & \\
\hline I-II & 1.000 & & & \\
\hline III-IV & $3.847(2.503-5.913)$ & & & \\
\hline FSIP status & & $<0.001$ & & 0.001 \\
\hline Negative & 1.000 & & 1.000 & \\
\hline Positive & $2.176(1.489-3.180)$ & & $1.876(1.274-2.764)$ & \\
\hline Ki67 status & & 0.006 & & 0.019 \\
\hline Negative & 1.000 & & 1.000 & \\
\hline Positive & $1.769(1.179-2.654)$ & & $1.629(1.082-2.453)$ & \\
\hline
\end{tabular}

Abbreviation, AC: adenocarcinoma; FSIP1: fibrous sheath interacting protein 1; SC: squamous carcinoma. 
advanced TNM stages and poorer prognosis. In addition, FSIP1-positive status was an independent prognostic factor for poor OS. To our best of knowledge, this is the first study to explore the role of FSIP1 in NSCLC.

FSIP1 is a component of the microtubule and dyneinrich fibrous sheath structure and may directly or indirectly support cell mitosis [3]. Indeed, Cappell et al. reported that FSIP1 depletion can enhance paclitaxel-induced mitotic arrest and/or the formation of micronucleated cells in NSCLC cell lines, and FSIP1-mediated alterations in microtubule and dynein function may support the microtubule network and enhance mitotic robustness in cancer cells [3]. In addition, FSIP1 can bind to and activate cancer/testis antigen proteins (including CABYR, SPA17, AKAP3, AKAP4, and ROPN1) in the fibrous sheath in tumor cells, in turn promoting cancer progression [3, 6-8]. These results are consistent with the association observed here between FSIP1-positive status and more advanced TNM stages and poorer prognosis in NSCLC. However, additional studies of the molecular mechanisms underlying the role of FSIP1 in NSCLC are required.

Ki67 levels, which are correlated with cancer cell proliferation and growth, are widely used in routine pathological examinations as a proliferation marker $[9,10]$. In addition, Ki67 is also used as a prognostic and diagnostic index for the evaluation of cancer biopsies, including lung cancer $[11,12]$. Our results confirmed that Ki67 was an independent prognostic factor in NSCLC (Table 2). We also used the c-index method to compare the prognostic capacities of Ki67 and FSIP1. The c-index value of FSIP1 was greater than that of Ki67, suggesting that FSIP1 had better prognostic capacity than Ki67. FSIP1 might therefore be particularly valuable during routine pathological examinations in NSCLC patients. However, it is worth noting that this study included only 202 NSCLC patients from a single institution; multicenter, large-scale studies are needed to confirm our results in NSCLC patients more generally. Additionally, due to limited data availability, we were not able to analyze the association between FSIP1 and the efficacy of adjuvant therapy in NSCLC; future studies are needed to evaluate that relationship as well.

We also compared the prognostic ability of FSIP1 in combination with the TNM staging system to the ability of the TNM staging system alone. The c-index for OS was greater for TNM+FSIP1 than for TNM staging alone, indicating that the addition of FSIP1 status improved the prognostic ability of the TNM staging system. Thus, FSIP1 may increase prognostic accuracy in NSCLC patients and might serve as a valuable supplementary index when used with the current TNM staging system.

In conclusion, we found that FSIP1 was highly expressed in NSCLC and was an independent prognostic factor in NSCLC patients. These results suggest that the evaluation of FSIP1 in combination with the current TNM staging system during routine examinations might help improve prognostic predictions in NSCLC patients.

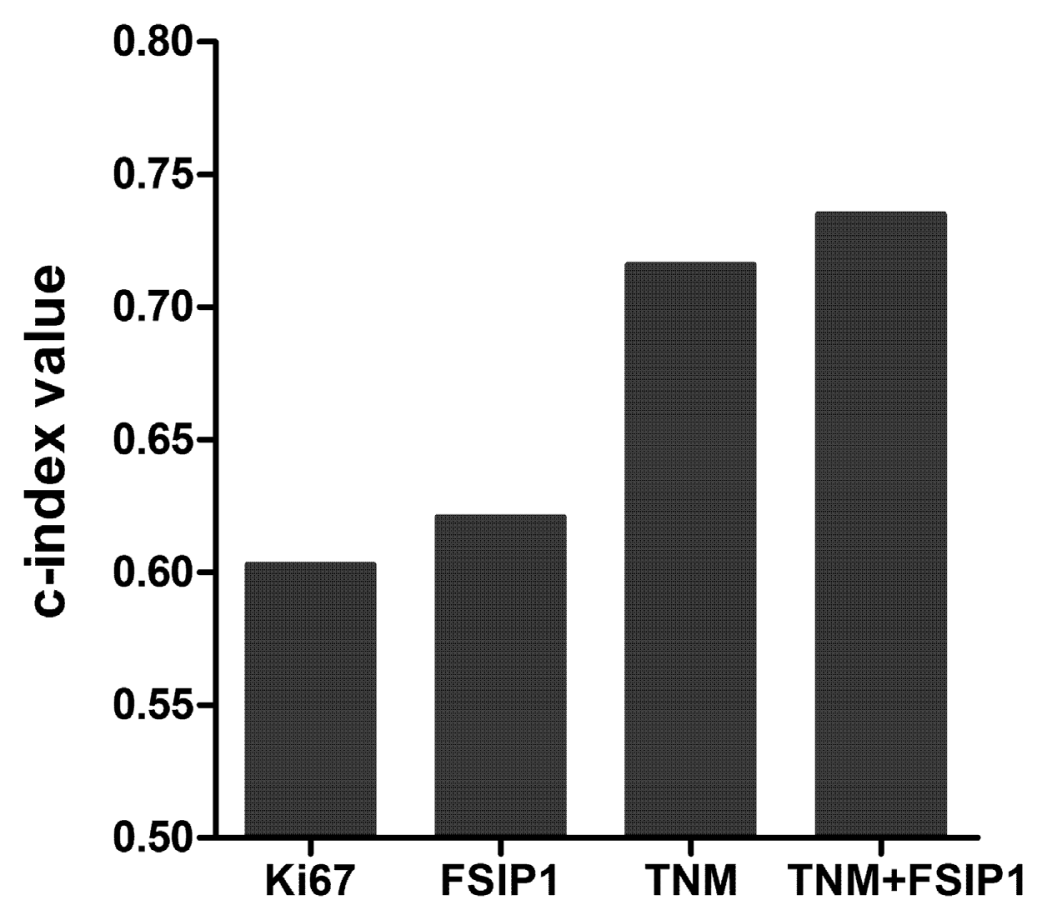

Figure 4: Comparison of c-index values for Ki67, FSIP1, TNM stage, and TNM+FSIP1. 


\section{MATERIALS AND METHODS}

\section{Patients and samples}

Primary NSCLC tissues and paired non-tumor adjacent tissues (NATs) were obtained from 202 patients who underwent lung cancer resection at Shengjing Hospital of China Medical University (Shenyang, China) between November 2009 and October 2013. Of these samples, 20 NSCLC tissues and paired NATs were assayed for FSIP1 mRNA and protein expression using real-time reverse transcription polymerase chain reaction (real-time PCR) and western blot, respectively. In addition, all 202 sample pairs were used for immunohistochemistry and included in prognosis analysis. Follow-up times ranged from 3 to 83 months with a median of 48 months. Tumor grades were staged according to 7th edition of the TNM staging system. The study was approved by the Research Ethics Committee of China Medical University. Written informed consent was obtained from all patients.

\section{RNA extraction, reverse transcription, and real- time PCR}

Total RNA was isolated from tissue samples using TRIzol reagent (Invitrogen Life Technologies, USA). Reverse transcription was conducted using the PrimeScript ${ }^{\mathrm{TM}} \mathrm{RT}$ reagent Kit with gDNA Eraser (Takara, China). Real-time PCR analyses were performed using SYBR Premix Ex Taq ${ }^{\mathrm{TM}}$ (Takara, China) on a Light Cycler 480 II Real-Time PCR system (Roche Diagnostics, Switzerland). The following primers were used: FSIP1, 5'-GTGTTCCCCCAGCTTTCCA-3' (forward) and 5'-TGCTTCAGTGACAAGAGCTTC-3' (reverse); GAPDH, 5'-CGGATTTGGTCGTATTGGG-3' (forward) and 5'-CTGGAAGATGGTGATGGGATT-3' (reverse). Relative FSIP1 expression was normalized to the GAPDH reference and calculated using the $2^{-\Delta \Delta \mathrm{CT}}$ method [13].

\section{Western blots}

Total protein was isolated using a protein extraction kit (ProMab, USA) followed by centrifugation. The protein content was quantitatively analyzed using a bicinchoninic acid protein assay and separated using $12 \%$ SDS-PAGE (sodium dodecyl sulfate polyacrylamide gel electrophoresis). The separated proteins were transferred to a PVDF (polyvinylidene fluoride) membrane (Millipore, USA). Samples were blocked in $5 \%$ bovine serum albumin for $2 \mathrm{~h}$ at room temperature and incubated with anti-FSIP1 (1:1000; Novus, USA) and anti-GAPDH (1:10000; Sigma, USA) primary antibodies as appropriate overnight at $4^{\circ} \mathrm{C}$. The membrane was washed in PBST and incubated with horseradish peroxidase-conjugated goat anti-rabbit IgG at room temperature for $1 \mathrm{~h}$. The membrane was washed with PBST again and the ECL kit was used for western blot detection. Relative FSIP1 protein levels compared to the GAPDH control were determined using ImageJ.

\section{Immunohistochemistry}

PV-9000 Polymer Detection System ImmunoHistological Staining (Zhongshan, Beijing, China) was used for immunostaining. Four- $\mu$ m-thick NSCLC tissue and NATs sections were obtained using a cryostat, deparaffinized with xylene, and rehydrated using a graduated ethanol series. In order to block endogenous peroxidase activity, tissue sections were initially incubated with $0.3 \%$ hydrogen peroxide solution for $10 \mathrm{~min}$. Sections were then incubated for $60 \mathrm{~min}$ at room temperature with rabbit polyclonal FSIP1 antibody (1:200 dilution, Novus, USA) and washed with phosphate buffered saline (PBS). Sections were then incubated with Polymer Helper at room temperature for $20 \mathrm{~min}$ and washed with PBS. Secondary polyperoxidase-antibody was then added and incubated at room temperature for $30 \mathrm{~min}$, followed by a final PBS wash. Diaminobenzidine was used as a chromogen to visualize staining. Negative control staining, in which the primary antibody was omitted, was conducted in parallel.

\section{Evaluation of immunohistochemical staining results}

The immunostaining results were evaluated by two pathologists independently using a semi-quantitative scoring system. Staining intensity values (0 for no staining; 1 for weak straining; 2 for moderate straining; and 3 for strong straining), and values representing the percentage of cells stained ( 0 for $\leq 5 \%$; 1 for $5-25 \%$; 2 for $25-50 \%$; 3 for $50-75 \%$; and 4 for $\geq 75 \%$ ) were assigned. These staining intensity and positive cell percentage values were multiplied to generate immunoreactivity scores (IS) [14]. Any scoring discrepancies were resolved by discussion. All tissue samples were then assigned to one of two groups based on IS; FSIP1-positive status was defined by detectable nuclear/cytoplasm immunoreactivity and an IS $\geq 4$ as determined by a receiver operating characteristic curve.

\section{Statistical analysis}

Continuous variables were analyzed using paired $t$-tests or non-parametric tests. Categorical variables were examined using the chi-square test. Survival rate was determined using the Kaplan-Meier method with log-rank tests. Univariate analysis was used to explore associations between prognostic factors and prognosis. Significant prognostic factors in univariate analysis were then analyzed in multivariate analyses using the Cox proportional hazards model. 
The predictive capacity of different models was evaluated by measuring discrimination, which is the ability to distinguish between high-risk and low-risk patients. Discrimination was quantified using Harrell's concordance index (c-index) $[15,16]$. A c-index value of 1.0 indicates a perfect discrimination; c-index values closer to 1.0 indicate more accurate predictive ability.

All data were analyzed using SPSS software version 20.0 and STATA software version 12.0. A two-tailed $p$ value $<0.05$ was considered statistically significant.

\section{Ethic statement}

The study was approved by the Research Ethics Committee of China Medical University. Written informed consent was obtained from all patients.

\section{ACKNOWLEDGMENTS}

We thank the Department of Thoracic Surgery of Shengjing Hospital of China Medical University for technical assistance.

\section{CONFLICTS OF INTEREST}

The authors declare no conflicts of interest.

\section{REFERENCES}

1. Chen W, Zheng R, Baade PD, Zhang S, Zeng H, Bray F, Jemal A, Yu XQ, He J. Cancer statistics in China, 2015. CA. 2016; 66:115-132.

2. Siegel RL, Miller KD, Jemal A. Cancer statistics, 2015. CA. 2015; 65:5-29.

3. Cappell KM, Sinnott R, Taus P, Maxfield K, Scarbrough M, Whitehurst AW. Multiple cancer testis antigens function to support tumor cell mitotic fidelity. Molecular and cellular biology. 2012; 32:4131-4140.

4. Chapman KB, Prendes MJ, Kidd JL, Sternberg H, West $\mathrm{MD}$, Wagner J. Elevated expression of cancer/testis antigen FSIP1 in ER-positive breast tumors. Biomarkers in medicine. 2013; 7:601-611.

5. Zhang H, Luo M, Jin Z, Wang D, Sun M, Zhao X, Zhao Z, Lei H, Li M, Liu C. Expression and clinicopathological significance of FSIP1 in breast cancer. Oncotarget. 2015; 6:10658-10666. doi: 10.18632/oncotarget.3381.
6. Brown PR, Miki K, Harper DB, Eddy EM. A-kinase anchoring protein 4 binding proteins in the fibrous sheath of the sperm flagellum. Biology of reproduction. 2003; 68:2241-2248.

7. Eddy EM, Toshimori K, O'Brien DA. Fibrous sheath of mammalian spermatozoa. Microscopy research and technique. 2003; 61:103-115.

8. Hofmann O, Caballero OL, Stevenson BJ, Chen YT, Cohen T, Chua R, Maher CA, Panji S, Schaefer U, Kruger A, Lehvaslaiho M, Carninci P, Hayashizaki Y, Jongeneel CV, Simpson AJ, Old LJ, et al. Genome-wide analysis of cancer/testis gene expression. Proceedings of the National Academy of Sciences of the United States of America. 2008; 105:20422-20427.

9. Li LT, Jiang G, Chen Q, Zheng JN. Ki67 is a promising molecular target in the diagnosis of cancer (review). Molecular medicine reports. 2015; 11:1566-1572.

10. Brown DC, Gatter KC. Ki67 protein: the immaculate deception? Histopathology. 2002; 40:2-11.

11. Scagliotti GV, Micela M, Gubetta L, Leonardo E, Cappia S, Borasio P, Pozzi E. Prognostic significance of Ki67 labelling in resected non small cell lung cancer. European journal of cancer. 1993; 29A:363-365.

12. Del Gobbo A, Pellegrinelli A, Gaudioso G, Castellani M, Zito Marino F, Franco R, Palleschi A, Nosotti M, Bosari S, Vaira V, Ferrero S. Analysis of NSCLC tumour heterogeneity, proliferative and 18F-FDG PET indices reveals Ki67 prognostic role in adenocarcinomas. Histopathology. 2016; 68:746-751.

13. Livak KJ, Schmittgen TD. Analysis of relative gene expression data using real-time quantitative PCR and the 2(-Delta Delta C(T)) Method. Methods. 2001; 25:402-408.

14. Pilch H, Weiss J, Heubner C, Heine M. Differential diagnosis of keratoacanthomas and squamous cell carcinomas: diagnostic value of DNA image cytometry and p53 expression. Journal of cutaneous pathology. 1994; 21:507-513.

15. Harrell FE, Jr., Lee KL, Mark DB. Multivariable prognostic models: issues in developing models, evaluating assumptions and adequacy, and measuring and reducing errors. Statistics in medicine. 1996; 15:361-387.

16. Newson RB. Comparing the predictive powers of survival models using Harrell's C or Somers' D. Stata J. 2010; 10:339-358. 